JOURNAL OF THE

AMERICAN MATHEMATICAL SOCIETY

Volume 18, Number 3, Pages 547-559

S 0894-0347(05)00480-7

Article electronically published on March 28, 2005

\title{
AN UNCOUNTABLE FAMILY OF NONORBIT EQUIVALENT ACTIONS OF $\mathbb{F}_{n}$
}

\author{
DAMIEN GABORIAU AND SORIN POPA
}

$\S 0$

Recall that two ergodic probability measure preserving (p.m.p.) actions $\sigma_{i}$ for $i=1,2$ of two countable groups $\Gamma_{i}$ on probability measure standard Borel spaces $\left(X_{i}, \mu_{i}\right)$ are orbit equivalent $(\mathrm{OE})$ if they define partitions of the spaces into orbits that are isomorphic, more precisely, if there exists a measurable, almost everywhere defined isomorphism $f: X_{1} \rightarrow X_{2}$ such that $f_{*} \mu_{1}=\mu_{2}$ and the $\Gamma_{1}$-orbit of $\mu_{1}$ almost every $x \in X_{1}$ is sent by $f$ onto the $\Gamma_{2}$-orbit of $f(x)$.

The theory of orbit equivalence, although underlying the "group measure space construction" of Murray and von Neumann [MvN36], was born with the work of H. Dye who proved, for example, the following striking result [Dy59: Any two ergodic p.m.p. free actions of $\Gamma_{1} \simeq \mathbb{Z}$ and $\Gamma_{2} \simeq \bigoplus_{j \in \mathbb{N}} \mathbb{Z} / 2 \mathbb{Z}$ are orbit equivalent.

Through a series of works, the class of groups $\Gamma_{2}$ satisfying Dye's theorem gradually increased until it achieved the necessary and sufficient condition: $\Gamma_{2}$ is infinite amenable OW80. In particular, all infinite amenable groups produce one and only one ergodic p.m.p. free action up to orbit equivalence (see also [CFW81] for a more general statement).

By using the notion of strong ergodicity, A. Connes and B. Weiss proved that any nonamenable group without Kazhdan property $(\mathrm{T})$ admits at least two non-OE p.m.p. free ergodic actions [CW80. The first examples of groups with uncountably many non-OE free ergodic actions was obtained in [BG81, using a somewhat circumstantial construction, based on prior work in [McD69. Within the circle of ideas brought up by Zimmer's cocycle super-rigidity [Zi84, certain Kazhdan property (T) lattices of Lie groups such as $\operatorname{SL}(n, \mathbb{Z}), n \geq 3$, were shown to admit uncountably many non-OE free ergodic actions as well (see GG88]). It is only recently that we learned that this property was shared by all infinite groups with Kazhdan property (T) $\mathrm{Hj}_{02}-\mathrm{b} \mid$, and thanks to another reason (bounded cohomology) by many torsion free finitely generated direct products $\Gamma_{1} \times \Gamma_{2}$, including nontrivial $(l \geq 2)$ products of free groups like $\mathbb{F}_{p_{1}} \times \mathbb{F}_{p_{2}} \times \cdots \times \mathbb{F}_{p_{l}}, p_{i} \geq 2$ MS02].

On the other hand, the situation for the free groups themselves or $\operatorname{SL}(2, \mathbb{Z})$ remained unclear and arouse the interest of producing more non-OE free ergodic actions of $\mathbb{F}_{n}$ than just the two given by [CW80] (more precisely in producing ways

Received by the editors September 12, 2003.

2000 Mathematics Subject Classification. Primary 37A20, 46L10.

The first author wishes to thank the C.N.R.S.

The second author was supported in part by NSF Grant 0100883.

(C)2005 American Mathematical Society Reverts to public domain 28 years from publication 
to distinguish them from the OE point of view), reaching the number 3 in 2001 [Po01] and then 4 in 2002 Hj02-b], for each finite $n \geq 2$.

Meanwhile, a certain rigidity phenomenon has been detected, behind the notion of cost Ga00 or $l^{2}$ Betti numbers Ga01, showing in particular that the rank of the free groups is an invariant of OE, as well as the virtual Euler characteristic of groups. As a consequence, all actions of finitely generated free groups were shown to have a trivial fundamental group (Ga00 or Ga01, Cor. 5.7]).

But these techniques have no hold on various actions of the same group. A new notion of rigidity for actions of a given group has been found in [Po01] (Def. 5.10.1), called relative property $(\mathrm{T})$. It is an $\mathrm{OE}$ invariant, is satisfied by certain actions of the free group $\mathbb{F}_{n}, 2 \leq n<\infty$, and not by others, and led to the already-mentioned third example by way of countability of its outer automorphism group (5.3.3 or 8.7 in [Po01]). This property will be crucial in our results and notice right away that it is satisfied by the natural actions of $\operatorname{SL}(2, \mathbb{Z})$ on the 2-torus $\mathbb{T}^{2}$ (cf. [Po01]). The main goal of this paper is to prove the following:

Theorem. For each $2 \leq n \leq \infty$, there exist uncountably many nonorbit equivalent actions of the free group $\mathbb{F}_{n}$, each being free ergodic and probability measure preserving on a standard Borel space.

Our result also applies to every virtually free group, and more generally to every group that is virtually a nontrivial free product of infinite amenable groups. All actions in our examples have the relative property $(\mathrm{T})$, thus having at most countable outer automorphism groups and countable fundamental group (trivial in the case of $\mathbb{F}_{n}$ ). Moreover, the uncountable family of actions can be taken so that any two of them are nonstably equivalent. Similar results are obtained for the associated $\mathrm{II}_{1}$ factors. Throughout the paper, we consider only standard Borel spaces.

$\S 1$

From the very beginning, the orbit equivalence theory has been strongly connected with operator algebras through the "group measure space construction" [MvN36, pp. 192-209], which was the first general way of producing various infinite dimensional von Neumann algebras (even before the "group von Neumann algebra" [MvN43 Sect. 5.3]): each ergodic p.m.p. free action $\alpha$ of an infinite countable group $\Gamma$ on a standard Borel space $(X, \mu)$ defines a type $\mathrm{II}_{1}$ von Neumann factor $M$ together with the abelian subalgebra $A=L^{\infty}(X, \mu) \subset L^{\infty}(X, \mu) \rtimes_{\alpha} \Gamma=M$ sitting in it as a Cartan subalgebra. Moreover two ergodic actions define isomorphic inclusions $\left(A_{1} \subset M_{1}\right) \simeq\left(A_{2} \subset M_{2}\right)$ iff they are orbit equivalent [FM77]. This fact establishes a useful equivalence between the study of actions of groups, up to orbit equivalence, and the study of the inclusions of Cartan subalgebras into $\mathrm{II}_{1}$ factors entailed by such actions, up to isomorphism of inclusions.

The notion of relative property $(\mathrm{T})$ for an action of a group in [P001] is in fact expressed in terms of its associated Cartan subalgebra, and the terminology used in this functional analytical framework is relatively rigid Cartan subalgebra (or rigid inclusion) $A \subset M$. Inspired by the notion of relative property (T) of Kazhdan-Margulis for inclusions of groups [Ma82] (see also dHV89 p. 16]) and by the Connes-Jones definition of property $(\mathrm{T})$ for $\mathrm{II}_{1}$ factors [CJ85, this property requires that whenever a completely positive map on $M$ (the operator algebra analogue of a positive definite function) is close to the identity on some "critical" finite subset of $M$, then it must be uniformly close to the identity on $A$. 
If a Cartan subalgebra $A \subset M$ comes from a group von Neumann algebra construction corresponding to an inclusion of groups $H \subset G=\Gamma \ltimes H$, then $A \subset M$ is rigid in the sense of Definition 4.2 in Po01] iff $H \subset G$ has the relative property (T) (cf. 5.1 in $[$ Po01] $)$. Since $\mathbb{Z}^{2} \subset \mathrm{SL}(2, \mathbb{Z}) \ltimes \mathbb{Z}^{2}$ has the relative property (T) (cf. Ma82]), this observation gives rise to an important example of an action with the relative property $(\mathrm{T})$, namely the standard action $\left(\mathrm{SL}(2, \mathbb{Z}), \mathbb{T}^{2}\right)$. More generally, M. Burger has shown that for any nonamenable subgroup of automorphisms of $\mathbb{Z}^{2}$, $\Gamma \subset \mathrm{SL}(2, \mathbb{Z})$, the induced inclusion $\mathbb{Z}^{2} \subset \Gamma \ltimes \mathbb{Z}^{2}$ has the relative property (T). So in fact by 5.1 in [Po01] all actions $\left(\Gamma, \mathbb{T}^{2}\right)$ have the relative property $(\mathrm{T})$. Equivalently, $A \subset M$ is a rigid inclusion of von Neumann algebras, where $M$ is the group von Neumann factor of the natural semidirect product $\Gamma \ltimes \mathbb{Z}^{2}$ and $A$ is the Cartan subalgebra $A=L^{\infty}\left(\mathbb{T}^{2}\right)$, identified via Fourier transform with the von Neumann algebra of the group $\mathbb{Z}^{2}$.

For the proof of the Theorem, we start with the above example of rigid inclusion $A \subset M$ in which $\Gamma=\mathbb{F}_{n}$. Then we use Dye's theorem to write the equivalence relation $\mathcal{R}_{1}$ implemented by the first of the generators of $\mathbb{F}_{n}$ (which one may assume acts ergodically) as a limit of an increasing one parameter family of ergodic hyperfinite equivalence relations $\mathcal{R}_{1, t}$, for $t \in(0,1]$. Together with $\mathbb{F}_{n-1}$, each of the $\mathcal{R}_{1, t}$ gives rise to a free ergodic action $\alpha_{t}$ of $\mathbb{F}_{n}$ on $\mathbb{T}^{2}$, with strictly increasing group measure space factors $A \subset M_{t} \subset M$ and $M_{t} \nearrow M$. By a rigidity property [Po01, 4.8], there is a value $c$ of the parameters after which $(c<t \leq 1)$ the actions $\alpha_{t}$ have relative property ( $\left.\mathrm{T}\right)$, equivalently, $A \subset M_{t}$ are rigid.

We then use the rigidity of each of these $A \subset M_{t}$ to prove that if an endomorphism of $M_{t}$ is close enough to the identity, then it has to be onto. This is done by analogy with the proof that $\operatorname{Out}\left(A \subset M_{t}\right)$ is countable in [Po01, 4.7], along the lines of Connes' pioneering rigidity result on the countability of $\operatorname{Out}(N)$ for $\mathrm{II}_{1}$ factors $N$ with the property (T) [Co80. Thus, by a separability argument as in Co80, Po86, if uncountably many inclusions $\left(A \subset M_{t}\right)$ are isomorphic, then one gets an endomorphism $M_{s} \simeq M_{t} \subset M_{s}$ close to the identity, thus onto, contradicting $M_{t} \neq M_{s}$. The partition of the interval of parameters $[c, 1]$ into the subsets where the factors $M_{t}$ are isomorphic is then composed of finite or countable subsets. We mention that in fact when proving the statement involving stable orbit equivalence, the above argument becomes slightly more complicated, with "isomorphism" (resp. "endomorphism") replaced by surjective (resp. nonsurjective) isomorphism between amplifications of suitable algebras.

$\S 2$

Set $I=(0,1) \cap \mathbb{Q}$ and $I_{t}=I \cap(0, t]$, for $t \in(0,1]$; set $G=\bigoplus_{i \in I} \mathbb{Z} / 2 \mathbb{Z}$ and $G_{t}=\bigoplus_{i \in I_{t}} \mathbb{Z} / 2 \mathbb{Z}$, for $0<t \leq 1$. This gives a strictly increasing continuum of subgroups of $G$.

Proposition 1. Let $\alpha$ be a free p.m.p. action of $\Gamma_{1} * \Gamma_{2}$, with $\Gamma_{1}$ an infinite amenable group acting ergodically. Then the following hold.

$1^{\circ}$. The action $\alpha$ is orbit equivalent to a free ergodic action $\sigma_{1}$ of the group $G * \Gamma_{2}$.

$2^{\circ}$. The restrictions $\sigma_{t}$ of $\sigma_{1}$ to the subgroups $G_{t} * \Gamma_{2}$ define a strictly increasing family of ergodic equivalence relations $\mathcal{S}_{t}$ indexed by $t \in(0,1]$, such that for any $s$ and for any increasing sequence $t_{n} \nearrow s$ one has $\mathcal{S}_{s}=\bigcup_{n} \mathcal{S}_{t_{n}}$.

$3^{\circ}$. The action $\sigma_{t}$ of $G_{t} * \Gamma_{2}$ is $O E$ to some ergodic free action $\alpha_{t}$ of $\Gamma_{1} * \Gamma_{2}$. 
$4^{\circ}$. The associated Cartan subalgebra inclusions define a strictly increasing family of subfactors $A \subset M_{t} \subset M_{1}, 0<t \leq 1$, such that $M_{t}=A \rtimes_{\alpha_{t}}\left(\Gamma_{1} * \Gamma_{2}\right), M_{1}=$ $A \rtimes_{\alpha}\left(\Gamma_{1} * \Gamma_{2}\right)$, and ${\overline{\bigcup_{t<s} M_{t}}}^{w}=M_{s}$, for each $s \in(0,1]$.

Lemma 2. Let $\Gamma=B * D$ and $\Lambda=C * D$ be free products such that $B$ and $C$ are infinite amenable groups. Every p.m.p. free action $\alpha$ of $\Gamma$, where $B$ acts ergodically, is orbit equivalent to a free action $\sigma$ of $\Lambda$, where (up to a null set) $\sigma_{\mid C}$ is any prescribed free ergodic p.m.p. C-action and $\sigma_{\mid D} \simeq \alpha_{\mid D}$.

Proof. By the Ornstein-Weiss OW80 and Dye Dy59 theorems, the free action of $B$ on $\left(X_{1}, \mu_{1}\right)$ is $\mathrm{OE}$ to the prescribed $C$-action on $\left(X_{2}, \mu_{2}\right)$, as witnessed by $f: X_{1} \rightarrow X_{2}$. Conjugating $\alpha_{\mid D}$ by $f$ leads to an action of the free product $C * D$, which is OE to $\alpha$. The equivalence relations defined by the actions, $\mathcal{S}_{1}=\mathcal{S}_{D}$ and $\mathcal{S}_{2}=\mathcal{S}_{B}=\mathcal{S}_{C}$, are in free product in the sense of [Ga00, Déf. IV.9], so that a nontrivial reduced word in the free product $C * D$ has obviously a set of fixed point of measure zero: the $\Lambda$-action $\sigma$ is free.

Proof of Proposition 1. By Lemma 2 $\alpha$ is orbit equivalent to a free action $\sigma_{1}$ of $G * \Gamma_{2}$, where the $G$-action is its Bernoulli shift on $\{0,1\}^{G}$. The restriction of $\sigma_{1}$ to $G_{t}$ remains ergodic, and the restriction $\sigma_{t}$ of $\sigma_{1}$ to $G_{t} * \Gamma_{2}$ is free ergodic. The properties of the family $\mathcal{S}_{t}$ are now obvious, and another application of Lemma 2 shows that each $\sigma_{t}$ is $\mathrm{OE}$ to a free action of $\Gamma_{1} * \Gamma_{2}$. The operator algebra statement $4^{\circ}$ follows immediately, due to the above and [FM77].

Recall that a Cartan subalgebra $A$ in a factor $M$ is a maximal abelian subalgebra whose normalizer $\mathcal{N}_{M}(A) \stackrel{\text { def }}{=}\left\{u \in \mathcal{U}(M) \mid u A u^{*}=A\right\}$ generates $M$ as von Neumann algebra (where $\mathcal{U}(M)$ is the group of unitary elements of $M$ ). Also, recall from 4.2 in $\mathrm{Po} 01$ that a Cartan subalgebra $A$ in a $\mathrm{II}_{1}$ factor $M$ is called relatively rigid if for any $\varepsilon>0$ there exist a finite set $F \subset M$ and $\delta>0$ such that if $\phi: M \rightarrow M$ is a completely positive map with $\tau \circ \phi \leq \tau, \phi(1) \leq 1$ and $\|\phi(x)-x\|_{2} \leq \delta, \forall x \in F$, then $\|\phi(a)-a\|_{2} \leq \varepsilon, \forall a \in A,\|a\| \leq 1$.

In [Po01, 4.7], it was proved that if $M$ is a type $\mathrm{II}_{1}$ factor with $A \subset M$ a relatively rigid Cartan subalgebra, then the subgroup of automorphisms $\mathcal{G}_{A} \subset \operatorname{Aut}(M)$ that are inner perturbations of automorphisms leaving $A$ pointwise fixed is open and closed in $\operatorname{Aut}(M)$, thus giving rise to a countable quotient group $\operatorname{Aut}(M) / \mathcal{G}_{A}$. In particular, if $A \subset M$ comes from a free ergodic p.m.p. action $\alpha$ of a group $\Gamma$ and $\mathcal{R}$ denotes the corresponding equivalence relation implemented by $\alpha$ on the probability space, then $\operatorname{Out}(\mathcal{R})$ is countable. The next proposition generalizes this result to the case of (not necessarily unital) endomorphisms of $M$ and of its amplifications.

Lemma 3. Let $M$ be a type $\mathrm{II}_{1}$ factor with $A \subset M$ a relatively rigid Cartan subalgebra. For each $x \in M$ denote $i(x)=x \oplus 0 \in \mathbb{M}_{2 \times 2}(M)$ and $p_{0}=i\left(1_{M}\right)=1 \oplus 0$. There exist $F \subset M$ finite and $\delta>0$ such that if $\theta: i(M) \rightarrow p \mathbb{M}_{2 \times 2}(M) p$ is a unital $*$-isomorphism satisfying $\|\theta(i(x))-i(x)\|_{2} \leq \delta, \forall x \in F$, for some projection $p \in \mathbb{M}_{2 \times 2}(M)$, then there exists $p^{\prime} \in \theta(i(A))^{\prime} \cap p \mathbb{M}_{2 \times 2}(M) p$ and a partial isometry $u \in \mathbb{M}_{2 \times 2}(M)$ such that

$$
u^{*} u=p_{0}, u u^{*}=p^{\prime}, \theta(i(M)) p^{\prime}=p^{\prime} \mathbb{M}_{2 \times 2}(M) p^{\prime}, \theta(i(a)) p^{\prime}=u i(a) u^{*}, \forall a \in A .
$$

If in addition $\theta(i(M))^{\prime} \cap p \mathbb{M}_{2 \times 2}(M) p=\mathbb{C} p$, then $\theta$ is surjective and $\tau(p)=1 / 2$, i.e., $\tau$ preserves the trace. 
Proof. Since $\theta$ is a unital $*$-isomorphism, it is completely positive. Thus, if $\phi$ : $M \rightarrow M$ is defined by $\phi(x)=p_{0} \theta(i(x)) p_{0}, x \in M$, then $\phi$ is completely positive and for all $x \in M$ we have

$$
\begin{aligned}
\|\phi(x)-x\|_{2} \leq & \|\theta(i(x))-i(x)\|_{2}+\left\|\theta(i(x))-p_{0} \theta(i(x)) p_{0}\right\|_{2} \\
\leq & \|\theta(i(x))-i(x)\|_{2}+\left\|\theta(i(x))-\theta(i(x)) p_{0}\right\|_{2} \\
& +\left\|\theta(i(x)) p_{0}-p_{0} \theta(i(x)) p_{0}\right\|_{2} \\
\leq & \|\theta(i(x))-i(x)\|_{2}+2\left\|\theta\left(p_{0}\right)-p_{0}\right\|_{2} .
\end{aligned}
$$

By the definition of relative rigidity it follows that there exist $F \subset M$ finite, with $1_{M} \in F$, and $\delta>0$, with $\delta \leq 1 / 8$, such that if a subunital subtracial completely positive map $\phi$ on $M$ satisfies $\|\phi(x)-x\|_{2} \leq \delta, \forall x \in F$, then $\|\phi(v)-v\|_{2} \leq 1 / 4$ for all elements $v$ in the unitary group $\mathcal{U}(A)$ of $A$. But then we also have

$$
\begin{aligned}
\|\theta(i(v))-i(v)\|_{2} & \leq\left\|\theta(i(v))-\theta(i(v)) p_{0}\right\|_{2}+\left\|\theta(i(v)) p_{0}-p_{0} \theta(i(v)) p_{0}\right\|_{2} \\
& \leq 2\left\|\theta\left(p_{0}\right)-p_{0}\right\|_{2}+\|\phi(v)-v\|_{2} \\
& \leq 2 \cdot 1 / 8+1 / 4=1 / 2 .
\end{aligned}
$$

Thus, if $h$ denotes the unique element of minimal Hilbert norm $\|\cdot\|_{2}$ in the weakly compact convex set $\overline{c 0}^{w}\left\{\theta(i(v)) i(v)^{*} \mid v \in \mathcal{U}(A)\right\} \subset p \mathbb{M}_{2 \times 2}(M) p_{0}$, then $\left\|h-p_{0}\right\|_{2} \leq$ 1/2. Also, since $\left\|\theta(i(v)) h i(v)^{*}\right\|_{2}=\|h\|_{2}, \forall v \in \mathcal{U}(A)$, by the uniqueness of $h$ it follows that $\theta(i(v)) h=h i(v), \forall v \in \mathcal{U}(A)$. By a standard functional calculus trick (as in group representations), if $u_{0} \in \mathbb{M}_{2 \times 2}(M)$ is the partial isometry in the polar decomposition of $h$, then $u_{0} \neq 0, u_{0} i(a)=\theta(i(a)) u_{0}, \forall a \in A$, and $u_{0}^{*} u_{0} \in$ $i(A)^{\prime} \cap i(M)=i(A),\left[u_{0} u_{0}^{*}, \theta(i(A))\right]=0$.

We can thus apply the above to $a=i^{-1}\left(u_{0}^{*} u_{0}\right) \in A$ to get $\theta\left(u_{0}^{*} u_{0}\right) u_{0}=u_{0} u_{0}^{*} u_{0}=$ $u_{0}$. But this implies $\theta\left(u_{0}^{*} u_{0}\right) u_{0} u_{0}^{*}=u_{0} u_{0}^{*}$, so that $\theta\left(u_{0}^{*} u_{0}\right) \geq u_{0} u_{0}^{*}$. Note that in particular this implies that $\theta$ does not "shrink" the trace $\tau$, i.e., $\tau(\theta(x)) \geq \tau(x), \forall x \in$ $p_{0} \mathbb{M}_{2 \times 2}(M) p_{0}$.

Since the normalizer $\mathcal{N}_{M}(A)$ of $A$ in $M$ acts ergodically on $A$, there exist partial isometries $v_{1}, v_{2}, \ldots, v_{n}$ in $M$ such that $v_{j} v_{j}^{*}, v_{j}^{*} v_{j} \in A, v_{j} A v_{j}^{*}=A v_{j} v_{j}^{*}, v_{j}^{*} v_{j} \leq$ $u_{0}^{*} u_{0}$ and $\sum_{j} v_{j} v_{j}^{*}=1$. Then $u=\sum_{j} \theta\left(i\left(v_{j}\right)\right) u_{0} i\left(v_{j}\right)^{*}$ is easily seen to be a partial isometry satisfying $u i(a)=\theta(i(a)) u, \forall a \in A$, with $u^{*} u=p_{0}, p^{\prime}=u u^{*} \in \theta(i(A))^{\prime} \cap$ $p \mathbb{M}_{2 \times 2}(M) p$. By spatiality it follows that $\theta(i(A)) p^{\prime}$ is a Cartan subalgebra (in particular maximal abelian) in $p^{\prime} \mathbb{M}_{2 \times 2}(M) p^{\prime}$.

Let $B=\theta(i(A))^{\prime} \cap p \mathbb{M}_{2 \times 2}(M) p$. Since $\theta(i(w))$ normalizes $\theta(i(A))$ for $w \in \mathcal{N}(A)$, it follows that $\theta(i(w))$ normalizes $B$ as well. Also, $\theta(i(A)) p^{\prime}$ maximal abelian implies $p^{\prime} B p^{\prime}=\theta(i(A)) p^{\prime}$. Thus, if $p_{1} \in B$ denotes the maximal abelian central projection of $B$, then $p_{1} \leq p^{\prime}$ and $\tau\left(p^{\prime}-p_{1}\right) \leq \tau\left(p-p^{\prime}\right)$. Since $\tau\left(p^{\prime}\right)=\tau\left(p_{0}\right)=1 / 2$ and

$$
\tau(p)-\tau\left(p_{0}\right) \leq\left\|p-p_{0}\right\|_{1} \leq 2\left\|p-p_{0}\right\|_{2} \leq 2 \delta \leq 1 / 4,
$$

it follows that $1 / 2-\tau\left(p_{1}\right) \leq 1 / 4$, so that $p_{1} \neq 0$. But since $B$ is normalized by $\theta(i(\mathcal{N}(A))), p_{1}$ is left invariant by $\operatorname{Ad}\left(w^{\prime}\right)$ for $w^{\prime} \in \theta(i(\mathcal{N}(A)))$. Thus, $\left[p_{1}, \theta(i(M))\right]=0$, so in particular $a \mapsto a p_{1}, a \in \theta(i(A))$ is an isomorphism. But $p^{\prime} B p^{\prime}=\theta(i(A)) p^{\prime} \simeq A$ as well. Thus, $p_{1}=p^{\prime}$.

In particular, this shows that if we denote $\theta_{0}(x)=u^{*} \theta(x) u, x \in M$, then $\theta_{0}$ is an isomorphism. Moreover, $\theta_{0}(a)=a, \forall a \in A$. Then we get

$$
w a w^{*}=\theta_{0}\left(w a w^{*}\right)=\theta_{0}(w) \theta_{0}(a) \theta_{0}\left(w^{*}\right)=\theta_{0}(w) a \theta_{0}\left(w^{*}\right)
$$


for all $a \in A$, implying that $\theta_{0}(w)^{*} w \in A^{\prime} \cap M=A$. Hence, $w \in \theta_{0}(w) A$. This shows that $\mathcal{N}_{M}(A) \subset \theta_{0}(M) A=\theta_{0}(M)$, so that $M \subset \theta_{0}(M)$, thus $\theta_{0}(M)=M$. But this implies $\theta(M)=M$ as well.

To state the next result, recall from $\mathrm{MvN43}$ that if $M$ is a $\mathrm{II}_{1}$ factor and $s>0$, then the $s$-amplification of $M$ is the $\mathrm{II}_{1}$ factor $M^{s}=p \mathbb{M}_{n \times n}(M) p$, where $n$ is some integer $\geq s$ and $p$ is a projection in $\mathbb{M}_{n \times n}(M)$ of (normalized) trace equal to $s / n$. This factor is defined up to isomorphisms implemented by unitary elements in large enough matrix algebras over $M$. Note also that in case $M$ has a Cartan subalgebra $A \subset M$, then $p$ can be taken in the Cartan subalgebra $\bigoplus^{n} A$ of $\mathbb{M}_{n \times n}(M)$ given by diagonal elements with entries in $A$ and $A^{t}=p\left(\bigoplus^{n} A\right) p$ is a Cartan subalgebra of $M^{t}$.

Two factors of type $\mathrm{II}_{1}, M_{1}, M_{2}$ are stably isomorphic if there exist $s>0$ such that $M_{1}$ is isomorphic to $M_{2}^{s}$. We then write $M_{1} \cong M_{2}$, while the symbol $\simeq$ will be used to denote usual isomorphism. Recall that the fundamental group of $M$ is the subgroup $\mathcal{F}(M):=\left\{s: M^{s} \simeq M\right\}$ of $\mathbb{R}_{+}^{*}$.

Proposition 4. Let $M$ be a type $\mathrm{II}_{1}$ factor with a relatively rigid Cartan subalgebra $A \subset M$. Then the fundamental group of $M$ is at most countable. If in addition $A \subset M_{t} \subset M, t \in(0,1]$, is a strictly increasing family of subfactors such that $M=\overline{\bigcup_{t<1} M_{t}}$, then we have the following.

$1^{\circ}$. There exists $c<1$ such that for all $c \leq t \leq 1, A \subset M_{t}$ are relatively rigid Cartan subalgebras.

$2^{\circ}$. If $c$ satisfies $1^{\circ}$, then for each $t \in[c, 1]$ the class of stable isomorphisms $\left\{s \mid M_{s} \cong M_{t}\right\}$ is at most countable.

Thus, the quotient set of parameters $[c, 1] / \sim$ is uncountable.

Proof. Item $1^{\circ}$ is part of $4.5 .4^{\circ}$ in [Po01]. To prove $2^{\circ}$, assume there exist $t_{0} \in$ $[c, 1]$ and an uncountable subset $L \subset[c, 1]$ such that $\theta_{t}: M_{t_{0}} \rightarrow M_{t}^{s(t)}, \forall t \in L$, for some $s(t)>0$ and some surjective isomorphisms $\theta_{t}$. We may further assume $n_{0}^{-1} \leq s(t) \leq n_{0}, \forall t \in L$, for some integer $n_{0} \geq 1$.

Let $F \subset M_{t_{0}}, \delta>0$ be such that if $\theta$ is a unital isomorphism of $i\left(M_{t_{0}}\right)=M_{t_{0}} \oplus 0$ into $p \mathbb{M}_{2 \times 2}\left(M_{t_{0}}\right) p$ satisfying $\theta\left(i\left(M_{t_{0}}\right)\right)^{\prime} \cap p \mathbb{M}_{2 \times 2}\left(M_{t_{0}}\right) p=\mathbb{C} p$ and $\|\theta(i(x))-i(x)\|_{2} \leq$ $\delta, \forall x \in F$, as in Lemma 3, then $\theta$ is onto and preserves the trace (cf. Lemma 3). By isomorphism, it follows that for each $t \in L$ we have: If $\theta$ is a unital isomorphism of $i\left(M_{t}^{s(t)}\right)$ into $p \mathbb{M}_{2 \times 2}\left(M_{t}^{s(t)}\right) p$ with range having trivial relative commutant and satisfying $\|\theta(i(x))-i(x)\|_{2} \leq \delta, \forall x \in \theta_{t}(F)$, then $\theta$ is surjective and preserves the trace.

We will now regard the isomorphisms $\theta_{t}, t \in L$, as isomorphisms of $M_{t_{0}} \oplus 0 \subset$ $M \oplus 0$ into $\mathbb{M}_{n_{0} \times n_{0}}(M)$, in the obvious way, where $M \oplus 0$ is the algebra of $n_{0} \times n_{0}$ matrices of the form $x \oplus 0$, having $x \in M$ as upper left entry and 0 elsewhere.

By the separability of the Hilbert space $L^{2}\left(\mathbb{M}_{n_{0} \times n_{0}}(M), \tau\right)$ and the uncountability assumption on $L$, it follows that there exist $t_{1} \neq t_{2} \in L$ such that $\| \theta_{t_{1}}(x)-$ $\theta_{t_{2}}(x) \|_{2} \leq \delta / n_{0}^{2}, \forall x \in F$. Assume $t_{1}<t_{2}$. Denote $\theta=\theta_{t_{1}} \circ \theta_{t_{2}}^{-1}: M_{t_{2}}^{s\left(t_{2}\right)} \simeq M_{t_{1}}^{s\left(t_{1}\right)} \subset$ $M_{t_{2}}^{s\left(t_{1}\right)}$ and note that for $\delta \leq 1 / 8$ we may assume $\theta$ is an isomorphism of $i\left(M_{t_{2}}^{s\left(t_{2}\right)}\right)$ into $\mathbb{M}_{2 \times 2}\left(M_{t_{2}}^{s\left(t_{2}\right)}\right)$ satisfying $\|\theta(i(x))-i(x)\|_{2} \leq \delta, \forall x \in \theta_{t_{2}}(F) \subset \theta_{t_{2}}\left(M_{t_{0}}\right)=$ $M_{t_{2}}^{s\left(t_{2}\right)}$. Note also that since $M_{t_{1}}^{\prime} \cap M_{t_{2}}=\mathbb{C}$, the range of $\theta$ has trivial relative commutant. 
By the above observation (consequence of Lemma 3) this implies $\theta$ is onto and trace preserving. Thus $s\left(t_{1}\right)=s\left(t_{2}\right)$ and $M_{t_{1}}=M_{t_{2}}$, a contradiction.

Finally, note that the above argument applied to just the factor $M$ and its amplifications shows that $\mathcal{F}(M)$ is countable.

For the next result recall that the fundamental group $\mathcal{F}(\mathcal{R})$ of an ergodic equivalence relation $\mathcal{R}$ is the set of all $t>0$ such that the $t$-amplification $\mathcal{R}^{t}$ of $\mathcal{R}$ is orbit equivalent to $\mathcal{R}$. Also, two equivalence relations $\mathcal{R}_{1}, \mathcal{R}_{2}$ are stably orbit equivalent $\left(\mathcal{R}_{1} \cong \mathcal{R}_{2}\right)$ if there exists $s>0$ such that $\mathcal{R}_{1}, \mathcal{R}_{2}^{s}$ are orbit equivalent. Note that if $M, M_{1}, M_{2}$ are the $\mathrm{II}_{1}$ factors associated to $\mathcal{R}, \mathcal{R}_{1}, \mathcal{R}_{2}$, respectively, then $\mathcal{F}(M) \supset \mathcal{F}(\mathcal{R})$ and $\mathcal{R}_{1} \cong \mathcal{R}_{2}$ implies $M_{1} \cong M_{2}$.

Corollary 5. For each $2 \leq n \leq \infty$ there exists an uncountable family of nonstably orbit equivalent free ergodic p.m.p. actions $\alpha_{t}$ of $\mathbb{F}_{n}$ such that moreover the corresponding equivalence relations $\mathcal{R}_{\alpha_{t}, \mathbb{F}_{n}}$ have at most countable fundamental group (trivial in the case $n<\infty$ ) and at most countable outer automorphism group.

Proof. For each $2 \leq n \leq \infty$, let $\mathbb{F}_{n} \subset \mathrm{SL}(2, \mathbb{Z})$ be a noncyclic free subgroup, and consider its standard action $\alpha$ on the 2 -torus $\mathbb{T}^{2}$. The following Lemma 6 gives a base of $\mathbb{F}_{n}$ with at least one ergodically acting element, thus allowing the use of Proposition 1 The family of Cartan subalgebra inclusions given by Proposition 1 satisfies Proposition 4 since $\alpha$ has the relative property (T), i.e., $A=L\left(\mathbb{Z}^{2}\right)=$ $L^{\infty}\left(\mathbb{T}^{2}, \lambda\right)$ is a relatively rigid Cartan subalgebra of $M_{1}=L\left(\mathbb{Z}^{2} \rtimes \mathbb{F}_{n}\right)=L\left(\mathbb{T}^{2}, \lambda\right) \rtimes_{\alpha}$ $\mathbb{F}_{n}$ (by Bu91 and Po01 5.1-5.2]). But nonisomorphic factors necessarily come from non-OE actions.

Thus, the uncountable family of nonstable equivalent actions, each one with a countable fundamental group, can be taken to be the $\alpha_{t}$ of Proposition 1, for a set of parameters representative of the classes $[c, 1] / \sim$ in Proposition $4\left(2^{\circ}\right)$. The triviality of the fundamental group in the case $n<\infty$ is a consequence of Ga01. Cor. 5.7]. The countability of the outer automorphism group follows from Po01. Cor. 4.7].

Lemma 6. If $\Gamma$ is a noncyclic free subgroup of $\mathrm{SL}(2, \mathbb{Z})$, then it admits a base of elements whose standard actions on the 2 -torus $\mathbb{T}^{2}$ are individually ergodic.

Proof. The criterion for an element to act ergodically is that it does not have a root of unity as eigenvalue or, equivalently, that it is hyperbolic when looking at its action on the circle, boundary of the hyperbolic disk. Fact: if $a$ and $b$ are two noncommuting elements of infinite order, where $a$ is parabolic, then there exists a power $k \in \mathbb{Z}$ such that $b^{k} a$ is hyperbolic: let $P_{a}$ be the unique fixed point of $a$, let $P_{b} \neq P_{a}$ be the first fixed point of $b$ met on the circle from the attracting side of $P_{a}$ and let $I$ be the corresponding interval between them (the case of a common unique fixed point is excluded by noncommutativity, then nonsolvability, of the subgroup generated by $a$ and $b$ ). Let $J$ be a closed interval strictly contained in $I$ with end point $P_{b}$. Then $a J$ is contained in the interior of $I$, and up to taking negative powers $P_{b}$ is attracting so that a big enough power $b^{k}$ satisfies $\left(b^{k} a\right) J \subset \operatorname{Int}(J)$, which is equivalent to hyperbolicity. Finally, starting from a base of $\Gamma$, one can successively transform it according to the fact so as to produce a base satisfying the lemma. 
For the next statement, recall that a group is virtually of a certain kind if it contains a finite index subgroup of that kind. The nontriviality hypothesis aims to avoid $\Lambda \simeq \Lambda *\{1\}$.

Corollary 7. Corollary 5 applies equally well with, instead of the group $\mathbb{F}_{n}$, any countable discrete group $\Gamma$ that is virtually a nontrivial free product $\Lambda$ of infinite amenable groups. More generally, if $\Gamma=\Lambda * H$ admits a free p.m.p. action $\alpha$ with the relative property $(\mathrm{T})$, where $H$ is infinite amenable and acts ergodically, then $\Gamma$ has an uncountable family of nonstably orbit equivalent free ergodic p.m.p. actions $\alpha_{t}$, such that $\mathcal{R}_{\alpha_{t}, \Gamma}$ have at most countable fundamental group (trivial in the case $\beta_{1}(\Lambda)$ is finite) and at most countable outer automorphism group.

Note that in order to ensure that the above action $\alpha$ has the relative property $(\mathrm{T})$, it is enough to get this property for some subgroup of $\Gamma$. This is, of course, trivial from the definition of the relative property $(\mathrm{T})$ ([P001, 4.6.2]).

Proof. The first part relies on the fact that actions of $\Gamma$ may be produced from actions of $\mathbb{F}_{n}$. Let $\Lambda$ be a finite index subgroup of $\Gamma$. Then the usual suspension construction associates, to each free p.m.p. action of $\Lambda$ on some standard Borel space $X$, a stably orbit equivalent free p.m.p. action of $\Gamma$ (given by right multiplication on itself) on the diagonal action quotient $\Lambda \backslash(X \times \Gamma)$. If the $\Lambda$-action is ergodic, then so is the $\Gamma$-action. Two non-OE $\Lambda$-actions lead to two non-OE $\Gamma$-actions.

By hypothesis, $\Lambda$ may be chosen to be a free product $H_{1} * H_{2} * \ldots$ of $n \in$ $\{2,3, \ldots, \infty\}$ infinite amenable groups. Start with an uncountable collection of free p.m.p. actions of $\mathbb{F}_{n}$ given by Corollary [5. Observe that in that construction one may assume that $\mathbb{F}_{n}$ is given with a base of elements that act individually in an ergodic manner.

Then up to removing at most countably many times a null set, as in Lemma 2 one can replace each cyclic factor in the decomposition of $\mathbb{F}_{n}$ by one $H_{i}$. This leads to uncountably many free p.m.p actions of $\Lambda$.

The last part is an immediate consequence of Propositions 1 and 4. Again, the triviality of the fundamental group in the case $\beta_{1}(\Lambda)<\infty$ follows from Ga01, Cor. 5.7].

We will now derive some applications of the above results to type $\mathrm{II}_{1}$ factors. To this end, recall from Section 6 in $\mathrm{Po01}$ that a Cartan subalgebra $A$ of a $\mathrm{II}_{1}$ factor $M$ is called $\mathrm{HT}_{s}$ if in addition to $A \subset M$ being a rigid inclusion, one has that $M$ has the property $H$ relative to $A$, i.e., there exists a sequence of unital completely positive $A-A$ bimodular maps $\phi_{n}$ on $M$ that tend to the identity in the point $\|\cdot\|_{2}$-topology and are compact relative to $A$ (cf. 2.1 in [Po01]). A $\mathrm{II}_{1}$ factor $M$ is in the class $\mathcal{H T}_{s}$ if it has such an $\mathrm{HT}_{s}$ Cartan subalgebra.

The property $\mathrm{H}$ of $M$ relative to $A$ is automatically satisfied whenever $M$ comes from a "group measure space construction", $M=L^{\infty}(X, \mu) \rtimes_{\alpha} \Gamma$, with $\Gamma$ a group satisfying Haagerup's compact approximation property and $\alpha$ a free ergodic measure preserving action of $\Gamma$ on the probability space $(X, \mu)$. Thus, since all subgroups $\Gamma$ of $\mathrm{SL}(2, \mathbb{Z})$ do have Haagerup's property (cf. [Ha79]) and $\left(\Gamma \ltimes \mathbb{Z}^{2}, \mathbb{Z}^{2}\right)$ has the relative property $(\mathrm{T})$ for all nonamenable $\Gamma \subset \mathrm{SL}(2, \mathbb{Z})$ (cf. Bu91), it follows by Lemma 6 that if $\Gamma=\mathbb{F}_{n}$ for some $2 \leq n \leq \infty$, then $A \subset M=L^{\infty}\left(\mathbb{T}^{2}, \lambda\right) \rtimes \Gamma$ is an $\mathrm{HT}_{s}$ Cartan subalgebra of the $\mathrm{HT}_{s}$ factor $M$. We then have the following corollary. 
Corollary 8. For each $2 \leq n \leq \infty$ there exists an uncountable family of mutually nonstably isomorphic II factors $M_{t}$ of the form $L^{\infty}(X, \mu) \rtimes \mathbb{F}_{n}$, all in the class $\mathcal{H} \mathcal{T}_{s}$. These factors have at most countable fundamental group (trivial in the case $n<\infty$ ). Moreover, in the case $n=\infty$ they can all be taken group von Neumann algebras of the form $L\left(\mathbb{F}_{\infty} \ltimes \mathbb{Z}^{2}\right)$, with $\mathbb{F}_{\infty}$ acting on $\mathbb{Z}^{2}$ as subgroups of automorphisms via various embeddings $\mathbb{F}_{\infty} \hookrightarrow \mathrm{SL}(2, \mathbb{Z})$.

Proof. By the above comments and [Po01], all actions $\alpha_{t}$ of $\mathbb{F}_{n}, 2 \leq n \leq \infty$, considered in the proof of Corollary 5, give rise to factors $M_{t}=L^{\infty}\left(\mathbb{T}^{2}, \lambda\right) \rtimes_{\alpha_{t}} \mathbb{F}_{n}$ in the class $\mathcal{H} \mathcal{T}_{s}$. By Proposition 4 , not only the actions $\alpha_{t}$ are not stably orbit equivalent (modulo countable sets), but even the factors $M_{t}$ are nonstably isomorphic and they all have a countable fundamental group. Since for $n<\infty$ by [8.6. $2^{\circ}$, Po01] they have the same $(\neq 0, \infty)$ first $\ell_{H T}^{2}$-Betti number, $\beta_{1}^{H T}\left(M_{t}\right)=n-1, \forall t$, the fundamental group $\mathcal{F}\left(M_{t}\right)$ is in this case trivial, $\forall t$.

To see that in the case of $n=\infty$ the factors $M_{t}$ can be taken group factors, write $\mathbb{F}_{\infty}$ as $\mathbb{F}_{I}$, the free group with a countable set of generators labeled by $I=(0,1) \cap \mathbb{Q}$, as in Proposition 1. Then let $I_{t}=I \cap(0, t]$, for $t \in(0,1]$, and denote by $\mathbb{F}_{I_{t}}$ the subgroup of $\mathbb{F}_{I}$ generated by generators with indices in $I_{t}$. Choose $\mathbb{F}_{I} \subset \mathrm{SL}(2, \mathbb{Z})$ an embedding of $\mathbb{F}_{I}$ as a group of automorphisms of $\mathbb{Z}^{2}$. By the remarks above again, the factors $L\left(\mathbb{F}_{I_{t}} \ltimes \mathbb{Z}^{2}\right)$ are in the class $\mathcal{H} \mathcal{T}_{s}$ and by Proposition 4 they all have countable fundamental group and are mutually nonstably isomorphic, modulo countable sets.

$\S 3$

The families of uncountably many nonequivalent free ergodic p.m.p. actions of $\mathbb{F}_{n}$ in the previous section are all obtained by approximating an initial equivalence relation $\mathcal{S}$ implemented by a relative property (T) action $\alpha$ of $\mathbb{F}_{n}$ from below by a one parameter family of subequivalence relations $\mathcal{S}_{t}$ implemented by actions $\alpha_{t}$ of the same group $\mathbb{F}_{n}$ : by the rigidity result (4.5 in $[\mathrm{Po} 01]$ ) the relations $\mathcal{S}_{t}$ (and thus the actions $\alpha_{t}$ ) have the relative property $(\mathrm{T})$ when close enough to $\mathcal{S}$, and thus Proposition 4 applies to obtain that modulo countable sets the $\alpha_{t}$ are nonequivalent (even nonstably orbit equivalent).

In this section we prove two more results in this vein. In both cases, from an initial relative property $(\mathrm{T})$ action of a group $\Gamma$ we will produce new actions having this property, but of groups which in general are different from the initial one. The first result is based on a construction similar to the one in Proposition 11. The second one gives a general hereditary property, showing that if an action $\sigma$ of a group $\Gamma_{0}$ has the relative property (T) and $\Gamma_{0}=\Gamma \rtimes G$ with $G$ amenable, then $\sigma \mid \Gamma$ has this property as well.

Proposition 9. Let $\alpha$ be a free p.m.p. action of $\Gamma_{1} * \Gamma_{2}$, with $\Gamma_{1}$ an infinite amenable group acting ergodically. There exists a strictly increasing family of ergodic equivalence relations $\mathcal{S}_{t}, t \in(0,1]$, implemented by free ergodic p.m.p. actions $\alpha_{t}$ of the group $\Lambda=\Gamma_{1} *\left(*_{j \in \mathbb{N}} \Gamma_{2}\right)$, such that the associated Cartan subalgebra inclusions define a strictly increasing family of subfactors $A \subset M_{t} \subset M_{1}, 0<t \leq 1$, with $M_{t}=A \rtimes_{\alpha_{t}} \Lambda, \forall t \in(0,1), M_{1}=A \rtimes_{\alpha}\left(\Gamma_{1} * \Gamma_{2}\right)$, and ${\overline{\bigcup_{t<s} M_{t}}}^{w}=M_{s}$, for each $s \in(0,1]$. If in addition $\alpha$ has the relative property $(\mathrm{T})$ (equivalently, if $A \subset M$ is rigid), then there exists $c<1$ such that $\alpha_{t}$ has the relative property $(T)$ 
(equivalently, $A \subset M_{t}$ is rigid) for all $t \in[c, 1]$, and thus the conclusions in part $2^{\circ}$ of Proposition 4 hold true.

Proof. We prove a series of statements similar to Proposition 10 keeping the hypotheses and the notation $I, I_{t}, G, G_{t}, \Gamma_{1}, \Gamma_{2}, \alpha, \sigma_{1}$ from there. For $t \in(0,1)$, define $\Lambda_{t}$ to be the kernel of the natural epimorphism $G * \Gamma_{2} \rightarrow G / G_{t} \simeq \bigoplus_{i \in I, i \notin I_{t}} \mathbb{Z} / 2 \mathbb{Z}$. Clearly, $G * \Gamma_{2}$ is the strictly increasing union of the normal subgroups $\Lambda_{t}, t \in(0,1)$. The restrictions $\tilde{\sigma}_{t}$ of the action $\sigma_{1}=\tilde{\sigma}_{1}$ to the subgroups $\Lambda_{t}$ are ergodic on $G_{t}$ and define a strictly increasing family of ergodic equivalence relations $\tilde{\mathcal{S}}_{t}$, with the same properties as $\mathcal{S}_{t}$.

On the other hand, $\Lambda_{t} \simeq G_{t} *\left(*_{j \in \mathbb{N}} \Gamma_{2}\right)$ : the quotient of the Bass-Serre tree of $G * \Gamma_{2}$ by $\Lambda_{t}$ is a graph of groups made of a set of vertices of degree 1, indexed by $g \in G / G_{t}$ with vertex groups $g^{-1} \Gamma_{2} g$, all connected to one "central" vertex with vertex group $G_{t}$. By Lemma $2 \tilde{\sigma}_{t}$ is OE to some ergodic free action $\tilde{\alpha}_{t}$ of $\Gamma_{1} *\left(*_{j \in \mathbb{N}} \Gamma_{2}\right)$.

Observe that, with the notation in the above proof, by normality of $\Lambda_{t}$, the outer automorphism group of $\mathcal{R}_{\tilde{\alpha}_{t}, \Lambda}$ contains $G / G_{t} \simeq \bigoplus_{n \in \mathbb{N}} \mathbb{Z} / 2 \mathbb{Z}$ for all $t \in[c, 1)$. Notice also that if we take $\Gamma_{1} \simeq \Gamma_{2} \simeq \mathbb{Z}$, then one has $\Lambda_{t} \simeq G_{t} * \mathbb{F}_{\infty}$ and $\Gamma_{1} *\left(*_{j \in \mathbb{N}} \Gamma_{2}\right) \simeq \mathbb{F}_{\infty}$.

Proposition 10. Let $\tilde{\Gamma}$ be a group of the form $\tilde{\Gamma}=H \ltimes \Gamma$, with $H$ amenable. Let $\sigma$ be a free p.m.p. action of $\tilde{\Gamma}$ on $(X, \mu)$. Then $\sigma$ has the relative property $(\mathrm{T})$ iff $\sigma_{\mid \Gamma}$ has this property.

Corollary 11. Let $\tilde{\Gamma}$ be a group of the form $\tilde{\Gamma}=H \ltimes \Gamma$, with $H$ amenable. Assume $\tilde{\Gamma}$ acts by outer automorphisms on an abelian group $\Delta$. Then the pair $(\Delta \ltimes \tilde{\Gamma}, \Delta)$ has the relative property (T) iff $(\Delta \ltimes \Gamma, \Delta)$ has this property.

Proof. By 5.1 in [Po01], it is sufficient to prove Proposition 10. Let $A=L^{\infty}(X, \mu)$, $\tilde{M}=A \rtimes_{\sigma} \tilde{\Gamma}, M=A \rtimes_{\sigma} \Gamma$. If $A \subset M$ is rigid, then $A \subset \tilde{M}$ is clearly rigid.

Conversely, assume $A \subset \tilde{M}$ is rigid and let $\tilde{F}=\tilde{F}(\varepsilon / 16) \subset \tilde{M}, \tilde{\delta}=\tilde{\delta}(\varepsilon / 16)>0$ be such that if $\tilde{\phi}$ is a completely positive map on $\tilde{M}$ with $\tau \circ \tilde{\phi} \leq \tau, \tilde{\phi}(1) \leq 1$ and $\|\tilde{\phi}(x)-x\|_{2} \leq \tilde{\delta}$ for any $x \in \tilde{F}$, then $\|\tilde{\phi}(a)-a\|_{2} \leq \varepsilon / 16, \forall a \in A,\|a\| \leq 1$.

By the amenability of $H$, it follows that there exists a finite subset $K \subset H$ such that

$$
\left\|\frac{1}{|K|} \sum_{g, h \in K} u_{g} E_{M}\left(u_{g}^{*} x u_{h}\right) u_{h}^{*}-x\right\|_{2} \leq \tilde{\delta} / 2, \forall x \in \tilde{F} .
$$

By using the amenability of $H$ again, by the Ornstein-Weiss theorem OW80 it follows that for any $\tilde{\delta}>0$ there exist a finite group $H_{0}=\bigoplus_{j}(\mathbb{Z} / 2 \mathbb{Z})_{j}$ and unitary elements $\left\{v_{g}\right\}_{g \in H_{0}}$ in the normalizer of $A$ in $A \rtimes H \subset \tilde{M}$ which implement a free action of $H_{0}$ on $(X, \mu)$ such that each $u_{g}, g \in K$, is $\varepsilon / 64$-close to an element $u_{g}^{\prime}$ in the normalizer of $A$ in $A \vee\left\{v_{g}\right\}_{g \in H_{0}} \simeq A \rtimes H_{0}$.

Thus, if we denote by $A_{0}=A \cap\left\{v_{g}\right\}_{g}^{\prime}$ the fixed point algebra of the action of $H_{0}$ on $A$ implemented by the unitaries $v_{g}$, then $A$ can be decomposed as $A=A_{0} \otimes A_{1}$ with $A_{1}$ generated by unitary elements $w_{h}, h \in \hat{H}_{0}$, satisfying $v_{g} w_{h} v_{g}^{*}=h(g) w_{h}$, $\forall g \in H_{0}, h \in \hat{H}_{0}$.

There exists $\delta>0$ such that if $\phi$ is a completely positive map on $M$ with $\tau \circ \phi \leq \tau$, $\phi(1) \leq 1$ and $\left\|\phi\left(w_{h}\right)-w_{h}\right\|_{2} \leq \delta, \forall h \in \hat{H}_{0}$, then $\left\|\phi\left(u w_{h}\right)-\phi(u) w_{h}\right\|_{2} \leq \varepsilon / 16, \forall u \in$ 
$\mathcal{U}(A), h \in \hat{H}_{0}$ (see, e.g., Corollary 1.1.2 in $[\overline{\mathrm{Po} 01}]$ ). Thus, for $u \in \mathcal{U}\left(A_{0}\right), h \in \hat{H}_{0}$ we have

$$
\begin{gathered}
\left\|\phi\left(u w_{h}\right)-u w_{h}\right\|_{2} \leq \\
\leq \varepsilon \phi\left(u w_{h}\right)-\phi(u) w_{h}\left\|_{2}+\right\| \phi(u)-u \|_{2} \\
\leq \varepsilon /\|\phi(u)-u\|_{2} .
\end{gathered}
$$

Moreover, $\delta$ can be chosen small enough so that we also have

$$
\left\|\phi\left(E_{M}\left(u_{g}^{*} x u_{g^{\prime}}\right)\right)-E_{M}\left(u_{g}^{*} x u_{g^{\prime}}\right)\right\|_{2} \leq(2|K|)^{-1} \tilde{\delta}, \forall g, g^{\prime} \in K .
$$

We set $F(\varepsilon)=\left\{E_{M}\left(u_{g}^{*} x u_{g^{\prime}}\right) \mid x \in \tilde{F}(\varepsilon / 16), g, g^{\prime} \in K\right\} \cup\left\{w_{h} \mid h \in \hat{H}_{0}\right\}, \delta(\varepsilon)=$ $\min \{\delta, \tilde{\delta}, \varepsilon / 4\}$ and will show that if $\phi$ is a completely positive map on $M$ such that $\tau \circ \phi \leq \tau, \phi(1) \leq 1$ and $\|\phi(x)-x\|_{2} \leq \delta(\varepsilon), \forall x \in F(\varepsilon)$, then $\|\phi(a)-a\|_{2} \leq \varepsilon, \forall a \in A$, $\|a\| \leq 1$.

To see this, note first that if we put $\tilde{\phi}(x) \stackrel{\text { def }}{=}|K|^{-1} \sum_{\tilde{N}^{\prime}, g^{\prime} \in K} u_{g} \phi\left(E_{\tilde{\varphi}_{M}}\left(u_{\tilde{g}^{*}}^{*} x u_{g^{\prime}}\right)\right) u_{g^{\prime}}^{*}$ for $x \in \tilde{M}$, then $\tilde{\phi}$ is a completely positive map on $\tilde{M}$ with $\tau \circ \tilde{\phi} \leq \tau, \tilde{\phi}(1) \leq 1$. Moreover, by (1) and (3), for each $x \in \tilde{F}(\varepsilon / 16)$ we have the inequalities

$$
\begin{gathered}
\|\tilde{\phi}(x)-x\|_{2} \leq|K|^{-1} \sum_{g, g^{\prime} \in K}\left\|u_{g} \phi\left(E_{M}\left(u_{g^{*}}^{*} x u_{g^{\prime}}\right)\right) u_{g^{\prime}}^{*}-u_{g} E_{M}\left(u_{g}^{*} x u_{g^{\prime}}\right) u_{g^{\prime}}^{*}\right\|_{2} \\
+\left\||K|^{-1} \sum_{g, g^{\prime} \in K} u_{g} E_{M}\left(u_{g}^{*} x u_{g^{\prime}}\right) u_{g^{\prime}}^{*}-x\right\|_{2} \leq \tilde{\delta} / 2+\tilde{\delta} / 2=\tilde{\delta} .
\end{gathered}
$$

Thus, $\|\tilde{\phi}(a)-a\|_{2} \leq \varepsilon / 16, \forall a \in A,\|a\| \leq 1$. Since for $a \in A$ we have

$$
\tilde{\phi}(a)=|K|^{-1} \sum_{g \in K} u_{g} \phi\left(E_{M}\left(u_{g}^{*} a u_{g}\right)\right) u_{g}^{*}=|K|^{-1} \sum_{g \in K} u_{g} \phi\left(\sigma_{g}^{-1}(a)\right) u_{g}^{*}
$$

and $\left\|u_{g}-u_{g}^{\prime}\right\|_{2} \leq \varepsilon / 64$, this implies that for $a_{0} \in A_{0},\left\|a_{0}\right\| \leq 1$ we have

$$
\begin{aligned}
\left\||K|^{-1} \sum_{g \in K} u_{g}^{\prime} \phi\left(a_{0}\right) u_{g}^{\prime *}-a_{0}\right\|_{2} & \leq\left\|\tilde{\phi}\left(a_{0}\right)-a_{0}\right\|_{2}+4\|K\|^{-1} \sum_{g \in K}\left\|u_{g}-u_{g}^{\prime}\right\|_{2} \\
& \leq \varepsilon / 16+\varepsilon / 16=\varepsilon / 8 .
\end{aligned}
$$

By convexity, since $\left\|u_{g}^{\prime} \phi\left(a_{0}\right) u_{g}^{\prime *}\right\|_{2}=\left\|\phi\left(a_{0}\right)\right\|_{2} \leq\left\|a_{0}\right\|$, it follows that for each $a_{0} \in A_{0},\left\|a_{0}\right\| \leq 1$, there exists $g=g\left(a_{0}\right) \in K$ such that

$$
\left\|\phi\left(a_{0}\right)-a_{0}\right\|_{2}=\left\|u_{g}^{\prime} \phi\left(a_{0}\right) u_{g}^{\prime *}-a_{0}\right\|_{2} \leq \varepsilon / 8 .
$$

Thus, with the notation in 1.1.2 of [Po01], by using part $4^{\circ}$ of Lemma 1.1.2 in Po01, in the Hilbert $M$-bimodule $\mathcal{H}_{\phi}$ we have $\left\|\left[u_{0}, \xi_{\phi}\right]\right\|_{2} \leq 2\left\|\phi\left(u_{0}\right)-u_{0}\right\|_{2} \leq \varepsilon / 4$, $\forall u_{0} \in \mathcal{U}\left(A_{0}\right)$ and $\left\|\left[w_{h}, \xi_{\phi}\right]\right\|_{2} \leq \varepsilon / 4, \forall h \in \hat{H}_{0}$, as well. This implies $\xi_{\phi}$ is $\varepsilon / 2$-close to a vector $\xi \in \mathcal{H}_{\phi}$ that commutes with both $\mathcal{U}\left(A_{0}\right)$ and the group $\left\{w_{h}\right\}_{h}$.

Since $\mathcal{U}\left(A_{0}\right)$ and $\left\{w_{h}\right\}_{h}$ generate $A$, it follows that $\xi$ commutes with all the elements in $A$. Thus, $\left\|\left[a, \xi_{\phi}\right]\right\|_{2} \leq \varepsilon / 2, \forall a \in A,\|a\| \leq 1$. By part $2^{\circ}$ of Lemma 1.1.3 in Po01, this implies $\|\phi(a)-a\|_{2} \leq \varepsilon, \forall a \in A,\|a\| \leq 1$.

Final remarks. $1^{\circ}$. Note that Propositions 9 and 4 , taken together, provide another construction of uncountable families of nonstably orbit equivalent actions of $\mathbb{F}_{\infty}$. As concerning orbit equivalence, observe that it is in fact enough, by Ga00. Prop. II.6] and $\mathrm{Hj02- \textrm {a }}$, to get one free ergodic p.m.p. action of $\mathbb{F}_{\infty}$ with countable fundamental group to get uncountably many non-OE actions by restriction to Borel subsets of various measures. However, these actions are stably orbit equivalent. 
$2^{\circ}$. Note that in Proposition 10 we have actually proved a slightly more general statement, namely: Let $M$ be a finite von Neumann algebra with $A \subset M$ a relatively rigid Cartan subalgebra. Let $A \subset N \subset M$ be an intermediate von Neumann subalgebra such that $M=N \rtimes_{\sigma} H$ for some free action of an amenable group $H$ on $N$, with the property that $\sigma_{h}(A)=A, \forall h \in H$. Then $A$ is relatively rigid in $N$ as well. Related to this statement, it would be interesting to investigate the following question: Assuming $B \subset N \subset M$ is an inclusion of finite von Neumann algebras such that $M$ is amenable relative to $N$, in the sense of Definition 3.5.2 of [Po01], and $B \subset M$ is rigid, does it follow that $B \subset N$ is rigid as well?

$3^{\circ}$. Let $\mathcal{S}$ be the equivalence relation implemented by the action of $\operatorname{SL}(2, \mathbb{Z})$ on $\left(\mathbb{T}^{2}, \lambda\right)$. By [Bu91 and [Po01, any subequivalence relation $\mathcal{S}_{0}$ of $\mathcal{S}$ implemented by a nonamenable subgroup $\Gamma_{0} \subset \mathrm{SL}(2, \mathbb{Z})$ has the relative property $(\mathrm{T})$. By the rigidity result 5.4 in [Po01, Propositions 1, 4, 9 and 10, the class of subequivalence relations $\mathcal{S}_{0} \subset \mathcal{S}$ for which this still holds true gets considerably larger. The following problem thus seems quite natural: Do all nonamenable ergodic subequivalence relations $\mathcal{S}_{0}$ of $\mathcal{S}$ have the relative property $(\mathrm{T})$ ?

\section{ACKNOWLEDGMEnts}

We are grateful to É. Ghys for helpful comments concerning Lemma 6 and to B. Bekka for pointing out to us the results on relative property $(\mathrm{T})$ in [Bu91].

\section{REFERENCES}

[BG81] S. Bezuglyı̆, V. Golodets: Hyperfinite and II 1 actions for nonamenable groups, J. Funct. Anal. (1) 40, 30-44 (1981). MR0607589|(82i:46096)

[Bu91] M. Burger: Kazhdan constants for SL(3, Z), J. Reine Angew. Math., 413 (1991), 36-67. MR 1089795 (92c:22013)

[CFW81] A. Connes, J. Feldman, B. Weiss: An amenable equivalence relation is generated by a single transformation, Ergodic Theory Dynamical Systems 1 (1981), 431-450. MR.0662736 (84h:46090)

[CJ85] A. Connes, V.F.R. Jones: Property T for von Neumann algebras, Bull. London Math. Soc. 17 (1985), 57-62. MR0766450 (86a:46083)

[Co80] A. Connes: A type $I I_{1}$ factor with countable fundamental group, J. Operator Theory 4 (1980), 151-153. MR0587372 (81j:46099)

[CW80] A. Connes, B. Weiss: Property $T$ and asymptotically invariant sequences, Israel J. Math. (3) 37 (1980), 209-210. MR.0599454 (82e:28023a)

[dHV89] P. de la Harpe, A. Valette: "La propriété T de Kazhdan pour les groupes localement compacts", Astérisque 175 (1989). MR1023471 (90m:22001)

[Dy59] H. Dye: On groups of measure preserving transformations, I, Amer. Math. J. 81 (1959), 119-159. MR0131516 (24:A1366)

[FM77] J. Feldman, C.C. Moore: Ergodic equivalence relations, cohomology, and von Neumann algebras I, II, Trans. Amer. Math. Soc. 234 (1977), 289-324, 325-359. MR0578656 (58:28261a) MR0578730(58:28261b)

[Ga00] D. Gaboriau: Coût des relations d'équivalence et des groupes, Invent. Math. 139 (2000), 41-98. MR1728876 (2001f:28030)

[Ga01] D. Gaboriau: Invariants $\ell^{2}$ de relations d'équivalence et de groupes, preprint 2001. Publ. Math. I.H.É.S. (1) 95 (2002), 93-150. MR1953191 (2004b:22009)

[GG88] S. L. Gefter, V. Ya. Golodets: Fundamental groups for ergodic actions and actions with unit fundamental groups, Publ. Res. Inst. Math. Sci., (6) 24 (1988, 1989), 821-847. MR.1000122 (91e:46090)

[Ha79] U. Haagerup: An example of non-nuclear $C^{*}$-algebra which has the metric approximation property, Invent. Math. 50 (1979), 279-293. MR0520930|(80j:46094)

[Hj02-a] G. Hjorth: A lemma for cost attained, UCLA preprint 2002.

[Hj02-b] G. Hjorth: A converse to Dye's Theorem, UCLA preprint, September 2002. 
AN UNCOUNTABLE FAMILY OF NONORBIT EQUIVALENT ACTIONS OF $\mathbb{F}_{n}$

[Ma82] G. Margulis: Finitely-additive invariant measures on Euclidian spaces, Ergodic. Th. and Dynam. Sys. 2 (1982), 383-396. MR0721730 (85g:28004)

[McD69] D. McDuff: Uncountably many II factors, Ann. of Math. (2) 90 (1969), 372-377. MR.0259625 (41:4261)

[MS02] N. Monod, Y. Shalom: Orbit equivalence rigidity and bounded cohomology, Preprint. (2002).

[MvN36] F. Murray, J. von Neumann: On rings of operators, Ann. Math. (2) 37 (1936), 116-229. MR 1503275

[MvN43] F. Murray, J. von Neumann: On rings of operators IV, Ann. Math. 44 (1943), 716-808. MR $0009096(5: 101 \mathrm{a})$

[OW80] D. Ornstein, B. Weiss: Ergodic theory of amenable group actions I. The Rohlin Lemma Bull. A.M.S. (1) 2 (1980), 161-164. MR0551753 (80j:28031)

[Po86] S. Popa: Correspondences, INCREST preprint 1986, unpublished.

[Po01] S. Popa: On a class of type $I_{1}$ factors with Betti numbers invariants, MSRI preprint 2001-024, revised math.OA/0209130, to appear in Ann. of Math.

[Zi84] R. Zimmer: Ergodic Theory and Semisimple Groups, Birkhauser, Boston, 1984. MR $0776417(86 \mathrm{j}: 22014)$

Umpa, UMR CNRS 5669, ENS-Lyon, F-69364 Lyon Cedex 7, France

E-mail address: gaboriau@umpa.ens-lyon.fr

Department of Mathematics, Univeristy of California, los Angeles, California 90095- 1555

E-mail address: popa@math.ucla.edu 